\title{
Erratum to: Pcal_0768, a hyperactive 4- $\alpha$-glucanotransferase from Pyrobaculum calidifontis
}

\author{
Sumaira Mehboob ${ }^{1} \cdot$ Nasir Ahmad $^{2} \cdot$ Naeem Rashid $^{1} \cdot$ Tadayuki Imanaka $^{3}$. \\ Muhammad Akhtar ${ }^{1,4}$
}

Published online: 18 July 2016

(C) Springer Japan 2016

\section{Erratum to: Extremophiles (2016) 20:559-566 DOI 10.1007/s00792-016-0850-x}

In the original publication the name of the organism was misspelled as "Pyrobacculum calidifontis" in the title of the manuscript. The correct name of the organism is "Pyrobaculum calidifontis".

The online version of the original article can be found under doi:10.1007/s00792-016-0850-x.

Naeem Rashid

naeemrashid37@hotmail.com; naeem.ff.sbs@pu.edu.pk

1 School of Biological Sciences, University of the Punjab, Quaid-e-Azam Campus, Lahore 54590, Pakistan

2 Institute of Agricultural Sciences, University of the Punjab, Quaid-e-Azam Campus, Lahore 54590, Pakistan

3 The Research Organization of Science and Technology, Ritsumeikan University, Kusatsu, Shiga 525-8577, Japan

4 School of Biological Sciences, University of Southampton, Southampton SO16 7PX, UK 